\title{
Nanocomposites incommensurables désordonnés
}

\author{
C. Ecolivet
}

\section{Groupe Matière Condensée et Matériaux, UMR 6626 du CNRS, Université de Rennes 1 , Rennes, France}

\section{I- Les matériaux}

Les matériaux composites sont constitués de différents composants et parmi ceux-ci on peut distinguer ceux qui cristallisent en deux sous-réseaux et, souvent il est facile de définir un sous réseau hôte et un sous réseau invité, il est alors possible de parler de matériaux nanovoire méso-poreux. Les structures résultent alors de l'équilibre entre les différentes interactions : hôte-hôte, hôte-invité et invité-invité. Suivant l'intensité relative des interactions on pourra avoir des structures qui pourront être fixées exclusivement par les interactions hôtehôte avec une géométrie et une taille des pores fixées à priori ou, sinon, dans le cas d'interactions hôte-invité non négligeables, les structures seront différentes pour des invités de taille et de nature différentes. En particulier dans ce cas, la structure du réseau hôte pur est différente de celle observée dans les différents sous-réseaux.

Ce type de matériau peut se rencontrer dans des matériaux minéraux et des matériaux moléculaires, toutefois, dans ces derniers, il est nécessaire de partir d'un sous-réseau hôte présentant une certaine rigidité mécanique. En effet, la stabilité des édifices cristallins moléculaires résulte très souvent d'interactions de van der Waals qui tendent à obtenir l'empilement le plus compact possible et, par conséquent, une structure poreuse n'aura que très peu de chances de pouvoir exister. Il est alors nécessaire d'utiliser des constituants développant des liaisons directionnelles. Dans les matériaux organiques une "brique de base" de ce jeu de construction supra moléculaire est la liaison hydrogène qui présente ce caractère de directionalité, bien que la force de la liaison, notablement inférieure à celle d'une liaison covalente, ne permette pas la stabilité de structures trop creuses. Les progrès de l'ingénierie moléculaire permettent l'obtention de pores de tailles différentes en utilisant des molécules jouant le rôle d'espaceurs comme dans les polymères ou les cristaux liquides[1].

Parmi les nanocomposites que l'on peut obtenir on trouve des structures bidimensionelles sous forme de plans stabilisés par des liaisons hydrogène avec des molécules 
intercalées entre ces plans ou des structures unidimensionnelles sous forme de canaux à l'intérieur desquels sont localisées les molécules invitées. Parmi ces dernières, le perhydrotriphénylène (PHTP) donne des structures dont la symétrie et la taille des canaux dépendent de la molécule invitée alors que les composés d'inclusion de l'urée présentent très souvent une phase hexagonale dont la taille est fixée par le sous-réseau de l'urée.

Plusieurs potentialités sont reconnues à ces structures hôte-invité et elles résultent du confinement des molécules invitées dans des orientations particulières. Cela permettrait, par exemple, d'aligner des molécules invitées présentant un dipôle permanent dans le même sens en écrantant les interactions avec dipôles voisins par l'intermédiaire des molécules hôtes, alors qu'à l'état cristallin de l'invité pur les dipôles permanents présentent très souvent des orientations antiparallèles. Cette stratégie d'assemblage permet d'obtenir de nouveaux matériaux avec des propriétés électro-optiques intéressantes mais d'autres applications intéressantes sont aussi envisageables [2]

\section{II- Incommensurabilité et désordre}

Lorsque suivant une des directions de l'espace le rapport des périodicités des mailles des deux sous-réseaux n'est pas dans un rapport de nombres entiers, il est alors irrationnel. On dit alors que le matériau est incommensurable, mais comme un nombre irrationnel est toujours compris entre deux nombres rationnels on ne sait jamais expérimentalement si on a affaire réellement à une incommensurabilité ou à une commensurabilité d'ordre élevé. Toutefois, les commensurabilités d'ordre élevé peuvent être pratiquement assimilées à une incommensurabilité car elles traduisent le même comportement physique. Parmi les systèmes de la famille hôte-invité, un des premiers à avoir été étudié de ce point de vue est le composé non stœchiométrique $\mathrm{Hg}_{3-\delta} \mathrm{AsF}_{6}$ qui présente des chaînes de mercure suivant deux directions perpendiculaires. La signature cristallographique de ces chaînes est un ensemble périodique de plans diffus de Bragg dans le diffractogramme. Le désordre régnant le long de ces chaînes entraîne un élargissement de ces plans variant comme le carré du vecteur d'onde de transfert si on suppose que la fonction de corrélation inter atomique est une gaussienne[3]. Cette signature des plans diffus élargis par le désordre se retrouve aussi dans les composés d'inclusion des alcanes dans l'urée[4] mais le caractère moléculaire de l'invité définit un facteur de forme pour la diffusion neutrons ou de Rayons $\mathrm{X}$ qui se manifeste, en particulier, par une diffusion diffuse plus large due à la périodicité de la chaîne d'alcane (plans $s$ ). Cette intensité diffuse a été analysée sur un spectromètre 3 axes (4F2) au LLB, et elle s'est révélée de nature essentiellement dynamique à température ambiante avec deux composantes inélastiques à $300 \mathrm{GHz}$ et $600 \mathrm{GHz}$, une composante élastique n'apparaissant qu'à plus basse température[5].

\section{III- Modulation et diffraction}

La présence de deux sous-réseaux indépendants de périodes différentes ne produirait que deux types de pics de Bragg reliés à ces sous-réseaux mais la stabilité du composite 
résulte de l'interaction de ses sous-réseaux qui exercent une modulation réciproque avec leur propre périodicité. Il en résulte des pics satellites supplémentaires qui ne peuvent s'indexer qu'avec quatre indices de Miller. Lorsque la modulation influe sur la position des atomes, pour simplifier nous ne considérerons qu'une direction appelée $\mathrm{z}$, les positions des atomes des sou-réseaux (1) et (2) s'écrivent :

$$
\mathrm{z}^{(1)}=<\mathrm{z}^{(1)}>+\mathrm{u}^{(1)}(<\mathrm{z}>) \quad \mathrm{z}^{(2)}=<\mathrm{z}^{(2)}>+\mathrm{u}^{(2)}(<\mathrm{z}>)
$$

avec: $\mathbf{u}^{(1)}(z)=u_{0}{ }^{(1)} \cos \left(2 \pi z / c_{2}+\phi_{0}{ }^{(1)}\right)$ et $u^{(2)}(z)=u_{0}{ }^{(2)} \cos \left(2 \pi z / c_{1}+\phi_{0}{ }^{(2)}\right)$. Les nouds dans l'espace réciproque sont repérés dans un espace à quatre dimensions, appelé super-espace, par les vecteurs $\mathbf{G}$ :

$$
\mathbf{G}=h \mathbf{a}^{*+} k \mathbf{b}^{*}+l_{1} \mathbf{c}_{1}{ }^{*}+l_{2} \mathbf{c}_{2}{ }^{*}
$$

$$
\text { Espace réel Espace réciproque }
$$

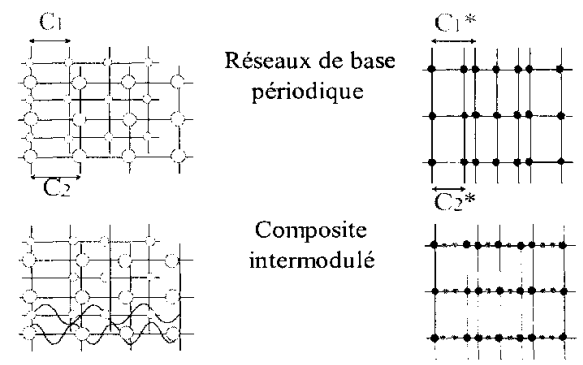

Fig 1: Modulation mutuelle des sous-réseaux et diffractogramme schématique dans le cas unidimensionnel

La description dans ce super-espace permet de faire réapparâtre la périodicité perdue à trois dimensions. Les pics indexés par (hk00) sont des pics de Bragg communs aux deux sous réseaux, ceux indexés par ( $\mathrm{hk10}$ ) ou ( $\mathrm{hk0 \textrm {m } )}$ proviennent d'un des sous réseaux et ceux indexés (hklm) sont des satellites prouvant l'incommensurabilité du système. Ces différents pics ont été observés tant en diffraction neutronique [6] qu'en diffraction de rayons $\mathrm{X}$ à l'ESRF sur le nonadécane/urée[7] et sur d'autres membres de la famille des alcanes/urée [4]. Ceci a été la preuve de l'appartenance de cette famille de composés aux structures composites incommensurables.

\section{IV- Dynamique des composites incommensurables}

Une première approche simpliste (approximation zéro) consisterait à décrire la dynamique de ces composés comme celle de deux sous-réseaux indépendants. Ainsi, dans un modèle unidimensionnel on représenterait les systèmes par deux chaînes monoatomiques de périodes différentes dans un rapport commensurable (approximant commensurable). Dans ce cas, on obtient bien sur deux branches de dispersion partant d'un vecteur d'onde nul et 
atteignant leur maximum pour différents vecteurs d'onde $\left( \pm \pi / \mathbf{c}_{1}{ }^{*}\right.$ et $\left.\pm \pi / \mathbf{c}_{2}{ }^{*}\right)$. On aurait ainsi deux vitesses du son longitudinales $V_{1}$ et $V_{2}$, une pour chaque sous réseau, ce qui bien, sur contraire aux lois de l'élasticité des solides. En fait l'interaction entre les deux sous réseaux se manifeste par une force de rappel entre ceux ci à vecteur d'onde nul dans un système commensurable, mais si le système est incommensurable un atome d'un sous réseau ne se retrouvera en aucun endroit du système avec le même voisinage et, dans un système infini, un sous réseau ressentira autant d'interactions de l'autre qui le tireront vers la droite que vers la gauche. Il en résultera une force moyenne nulle lors du déplacement d'ensemble d'un sous réseau par rapport à l'autre. Dans le cas ou l'intermodulation pourra être décrite par une fonction analytique il pourra exister un mode de vibration correspondant au glissement d'un sous réseau par rapport à l'autre qui aura une fréquence nulle pour un vecteur d'onde nul. Cela le fera ressembler à un mode acoustique mais ses déplacements correspondants seront ceux d'un mode optique en centre de zone, en particulier ce mode ne peut être directement excité par des capteurs ultrasonores. Evidemment tout ceci se modifiera au fur et à mesure que le vecteur d'onde prendra des valeurs plus grandes, et aux nœuds des sous réseaux on devrait retrouver les vitesses du son respectives des sous-réseaux. Traiter la dynamique de ces systèmes pour tous les vecteurs d'onde est très difficile, un modèle fructueux est un modèle de double chaîne dérivant du modèle de Frenkel-Kontorova où les interactions intra-chaînes sont modulées par la chaîne voisine[8]. Dans le cas d'une modulation analytique comme celle décrite au paragraphe précédent, on peut montrer que les vitesses du mode acoustique $V_{+}$et du mode de glissement $V$. peuvent s'exprimer en fonction des vitesses $V_{1}$ et $V_{2}$ ainsi que des masses linéiques des chaînes $\rho_{1}$ et $\rho_{2}$ :

$$
\mathrm{V}_{ \pm}{ }^{2}=\left(\mathrm{V}_{1}{ }^{2}+\mathrm{V}_{2}{ }^{2}\right) / 2\left\{1 \pm\left|\mathrm{V}_{1}{ }^{2}-\mathrm{V}_{2}{ }^{2}\right|\left|\rho_{1}-\rho_{2}\right| /\left(\mathrm{V}^{2}{ }_{1}+\mathrm{V}_{2}{ }^{2}\right)\left(\rho_{1}+\rho_{2}\right)\right.
$$

Si on applique cette formule à $\mathrm{Hg}_{3-\delta} \mathrm{AsF}_{6}$, où une branche de phonons prenant son origine aux réflexions de Bragg du mercure avait été détectée par diffusion cohérente inélastique de neutrons à Brookhaven [9], on est amené à comparer la pente de cette branche $3620 \mathrm{~m} / \mathrm{s}$ à la vitesse du son dans ce métal liquide qui est beaucoup plus basse $: 1450 \mathrm{~m} / \mathrm{s}$. En tenant compte du rapport des masses du mercure et $\mathrm{du}_{\mathrm{AsF}} \mathrm{As}_{6}$ on attribuerait alors cette branche observée au mode de glissement alors que le mode longitudinal acoustique présente une vitesse de $2130 \mathrm{~m} / \mathrm{s}$.

Dans le cas des composés d'inclusion des alcanes dans l'urée et, en particulier du nonadécane, certains auteurs avaient rapporté l'observation d'un mode acoustique supplémentaire observé par diffusion Brillouin dans l'heptadécane-urée[10] ], toutefois des expériences similaires de diffusion Brillouin dans un composite très voisin le nonadécane/urée n'ont pas confirmé cette observation mais elles on mis en évidence des composantes quasi élastiques dont une d'entre elles pourrait être liée directement à l'incommensurabilité de la structure [11]. Les mesures des propriétés élastiques par diffusion Brillouin ont montré que l'élasticité du composite était assez peu dépendante de la nature et de la taille des molécules invitées. Ceci laisse penser que les interactions entre molécules invitées sont faibles Des études par diffusion cohérente inélastique de neutrons n'ont pas réussi, à ce jour, à mettre en évidence la branche de phonons correspondant au mode de glissement, la manifestation de 
l'incommensurabilité semble se trouver dans l'amortissement anormal de la branche longitudinale acoustique émanant de la Bragg (1040) du sous réseau d'urée en accord avec les mesures de diffusion Brillouin suivant cette même direction.[12] Notons qu'à la différence des structures modulées, les diverses excitations sont théoriquement présentes aux différents nœuds de l'espace réciproque (Braggs et satellites)[13] sans toutefois pouvoir prédire, pour le moment, leurs intensités respectives pour un cristal réel.

\section{V- Transitions structurales dans les composites}

Il semble que le désordre inhérent à ces composés d'inclusion de l'urée limite l'observation des excitations spécifiques de telles structures d'autant plus qu'à basse température une transition de phase de type ferroélastique entraîne l'apparition de domaines qui provoquent l'éclatement de raies Bragg. Il est bien sur très difficile dans de telles conditions de réaliser des expériences de diffusion inélastique de neutrons.
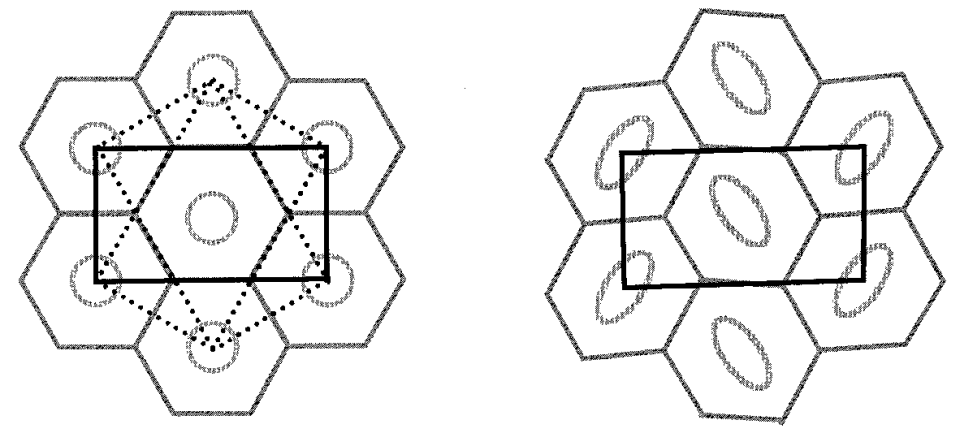

Fig 2 : Cisaillement de la structure lors du passage de la phase hexagonale désordonnée (à gauche) à la phase orthorhombique (à droite).

L'étude des transitions des instabilités structurales dans ces matériaux présente la particularité de faire passer la structure d'un groupe de superespace à un autre groupe de superespace avec la possibilité de retrouver les signatures de cette modification en des endroits différents de l'espace réciproque puisque les pics de diffraction de surstructure des sous réseaux seront positionnés différemment dans l'espace réciproque et que la dynamique de chaque sous réseau pourra elle aussi avoir ses particularités.

Dans le n-alcanes/urée la phase haute température hexagonale se transforme en une phase de symétrie orthorhombique à basse température caractérisée d'une part par un cisaillement du sous réseau d'urée dans le plan de base et d'autre part par une mise en ordre antiferro des molécules d'alcanes d'un canal à l'autre. Toutefois un désordre longitudinal suivant les canaux subsiste comme l'indique la persistance des plans diffus de type $s$. Un scénario pour cette transition structurale couple l'arrêt des réorientations des alcanes autour de leur axe long au cisaillement du réseau d'urée[14].Dans le nonadécane/urée des anomalies thermodynamiques ont été observées à une dizaine de $\mathrm{K}$ en dessous de la température de transition. Ces anomalies se retrouvent aussi bien dans des anomalies élastiues repérées par diffusion Brillouin que dans des intensités de surstructures qui se mettent à croître en dessous 
de cette température (Tc-10K). Ces anomalies laissent penser qu'il existe une phase intermédiaire dans cette gamme de température. L'application d'une pression hydrostatique déplace la température de transition vers les plus hautes températures d'environ $10 \mathrm{~K} / \mathrm{kbar}$ mais à des pressions plus élevées (4-5kbar) une autre phase apparaît.

\section{Conclusion}

Les propriétés physiques des composites sont potentiellement extraordinaires avec les possibilités quasi-infinies de l'ingénierie moléculaire et à ce titre ils méritent l'attention des physiciens. De plus ils présentent des transitions structurales originales et leur complexité intermédiaire entre les structures modulées et les quasicristaux permettent de tester des idées communes à ces structures.

Le concept de composite est bien sûr applicable aux composés minéraux qui en ont fournit le premier exemple étudié pour sa dynamique : $\mathrm{Hg}_{3-\delta} \mathrm{AsF}_{6}$ mais aussi à d'autres types de composés comme les supraconducteurs à haut Tc ou ceux du type « échelles de spin ». Malheureusement ces types de composés ont souvent un caractère métallique ou absorbent fortement la lumière et donc ne permettent pas des études conjointes diffusion neutroniquediffusion de la lumière qui seraient très profitables.

La notion de composite peut être même poussée plus loin : il est récemment apparu dans la littérature qu'un corps pur puisse présenter une structure composite où il est à la fois l'hôte et l'invité. Sous pression hydrostatique, des chaînes de strontium ou de baryum se trouvent incluses dans des canaux formés par ces mêmes atomes [15], c'est à partir de cette description que la phase $\mathrm{V}$ du strontium et du baryum ont pu être résolues. L'intermodulation des réseaux qu'implique la notion de composite incommensurable pourrait ainsi permettre d'expliquer l'origine de modulations incommensurables observées en introduisant la notion de sousréseaux et en la validant par l'observation de la dynamique caractéristique[16].

\section{Remerciements}

Cette présentation n'aurait pas été possible sans le concours des membres de l'équipe et les doctorants successifs du GMCM travaillant sur les structures apériodiques (B. Toudic, J.Etrillard, J. Ollivier, R. Lefort, H. Le Lann, L. Bourgeois) et des collaborations extérieures : F. Guillaume et M. Couzi du LPCM de l'université de Bordeaux, T. Breczewski de Bilbao, P. Bourges du L.L.B. Saclay et R. Currat de l'I.L.L. Grenoble

\section{Références}

1. M.J. Zaworotko Chem. Com. (2001)1-9 ; M. D. Ward, A. M. Pivovar; Current Opinion in Solid State and Materials Science 4 (1999) 581- 586

2 P. J. Langley and J. Hulliger; Chem. Soc. Rev., 1999, 28, 279-291

3 R. Spal, C.E. Chen, T. Egami, P.J. Nigrey and A.J. Heeger Phys. Rev. B 21 (8) 3110-3118 (1980)

4 T. Weber, H. Boysen and F. Frey Acta Cryst. (2000). B56, 132-141 
5 R. Lefort, B. Toudic, J. Etrillard, F. Guillaume, P. Bourges, R. Currat and T. Breczewski; Eur. Phys. J. B 24, $51\{57$ (2001)

6 R.Lefort,J.Etrillard,B.Toudic,F.Guillaume,T.Breczewski,P.Bourges, Phys.Rev.Lett.77(19) (1996);4027.

7 P. Rabiller communication privée

8 O. Radulescu, T. Janssen, Phys.Rev.B60 (1999) 12737

9 I.U. Heilmann,J.D. Axe,J.M. Hastings,G. Shirane, A.J. Heeger and A.J Mac Diarmid; Phys.RevB20(2)751-762 (1979)

10 D.Schmicker, S.vanSmaalen, J.L.deBoer, C.Haas, K.D.M.Harris,Phys.Rev.Lett.74(5) (1995) 34.

11 J. Ollivier, C. Ecolivet, S. Beaufils, F. Guillaume and T. Breczewski; Euro. Phys. Lett. 43 (1998) 546

12 R. Lefort (1998) Thèse de l'Université de Rennes1 (non publiée)

13 T. Janssen (2000) communication privée

14. R. M Lynden-Bell ; Molecular Physics 79 (1993)313

15 Mc Mahon et al P.R.B61 (2000) 3135

16 J. Etrillard, P. Bourges, H.F. He, B. Keimer, B. Liang, and C.T. Lin; Europhysics Letters, 55(2) 201-207(2001) 